An International Journal of Optimization and Control: Theories \& Applications ISSN:2146-0957 eISSN:2146-5703

Vol.8, No.2, pp.195-200 (2018)

http://doi.org/10.11121/ijocta.01.2018.00601

RESEARCH ARTICLE

\title{
A simulation algorithm with uncertain random variables
}

\author{
Hasan Dalman \\ Department of Computer Engineering, Istanbul Gelisim University, Turkey \\ hdalman@gmail.com
}

\begin{tabular}{|c|c|}
\hline ARTICLE INFO & ABSTRACT \\
\hline $\begin{array}{l}\text { Article History: } \\
\text { Received 05 April } 2018 \\
\text { Accepted 22 April } 2018 \\
\text { Available 25 April } 2018\end{array}$ & \multirow{2}{*}{$\begin{array}{l}\text { In many situations, uncertainty and randomness concurrently occur in a sys- } \\
\text { tem. Thus this paper presents a new concept for uncertain random variable. } \\
\text { Also, a simulation algorithm based on uncertain random variables is presented } \\
\text { to approximate the chance distribution using pessimistic value and optimistic } \\
\text { value. An example is also given to illustrate how to use the presented simula- } \\
\text { tion algorithm. }\end{array}$} \\
\hline $\begin{array}{l}\text { Keywords: } \\
\alpha \text { - optimistic value }\end{array}$ & \\
\hline
\end{tabular}

$\alpha$-pessimistic value

Uncertain random variables

Uncertainty theory

Simulation

AMS Classification 2010:

68T37, 03E72

\section{Introduction}

Liu [1] introduced the uncertain random variable for modeling complex systems. In other words; uncertain random variable is improved to illustrate the phenomenon which mixes uncertainty with randomness. If we receive historical data from the sample, we can estimate the probability distribution. But if we have a new product, we can not achieve the probability distribution of this new product owing to lack of data. In this case, we run across both randomness and human uncertainty. Human uncertainty is investigated by some scholars. As a branch of mathematics based on normality, duality, subadditivity and product axioms, uncertainty theory was introduced by Liu [2] in 2007. Gao [3] presented uncertain bimatrix game. Yang and Gao [4 studied uncertain differential game. Gao and Qin [5] introduced the degree connectivity of uncertain graph. Dalman [6] presented a model for the uncertain multi-item solid transportation problem. Dalman [7] constructed models of uncertain random multi-item solid transportation problem. To model uncertain random event, Liu [8] introduced the chance theory to networks optimization problem. Some scholars derived properties of uncertain random entropy [9, 10].

By employing chance theory, an uncertain random project scheduling problem is presented by Ke et al. [11. They introduced an uncertain random simulation which randomly produces the sample points. But, this algorithm is ambivalent due to produces different values at different time. A simulation algorithm is presented to solve uncertain random shortest path problem by Sheng and Gao [12].

Therefore this paper presents an algorithm which includes the inverse uncertainty distribution and uniformly produces the sample points. It has powerful performances on the reliability and stability than the algorithms in 11, 12. The paper is built as follows: some basic knowledge of uncertainty theory and chance theory is presented in section II. Section III shows some formulas for uncertain random variables presents an uncertain random simulation algorithm. A numerical example is presented in Section IV. Finally, this paper closes in Section V. 


\section{Preliminaries}

\subsection{Uncertainty theory}

Let $\Gamma$ be a nonempty set, $\mathcal{L}$ be a $\sigma$-algebra over $\Gamma$ and $\mathcal{M}$ be an uncertain measure. Then $(\Gamma, \mathcal{L}$, $\mathcal{M})$ is a measurable space. A set function $\mathcal{M}$ : $\mathcal{L} \rightarrow[0,1]$ is called an uncertain measure if it satisfies the following four axioms:

Axiom 1. (Normality Axiom)(Liu [2]): $\mathcal{M}\{\Gamma\}$ $=1$ for the universal set $\Gamma$.

Axiom 2. (Duality Axiom) $($ Liu [2] $): \mathcal{M}\{\Lambda\}+$ $\mathcal{M}\left\{\Lambda^{c}\right\}=1$ for any event $\Lambda$.

Axiom 3. (Subadditivity Axiom)(Liu [2]): For every countable sequence of events $\Lambda_{1}, \Lambda_{2}, \cdots$, we have

$$
\mathcal{M}\left\{\bigcup_{i=1}^{\infty} \Lambda_{i}\right\} \leq \sum_{i=1}^{\infty} \mathcal{M}\left\{\Lambda_{i}\right\}
$$

Axiom 4. (Product Axiom)(Liu [13]): Let $\left(\Gamma_{k}, \mathcal{L}_{k}, \mathcal{M}_{k}\right)$ be uncertainty spaces for $k=1,2$, ... The product uncertain measure $\mathcal{M}$ is an uncertain measure satisfying

$$
\mathcal{M}\left\{\prod_{k=1}^{\infty} \Lambda_{k}\right\}=\bigwedge_{k=1}^{\infty} \mathcal{M}_{k}\left\{\Lambda_{k}\right\}
$$

where $\Lambda_{k}$ are arbitrarily chosen events from $\mathcal{L}_{k}$ for $k=1,2, \cdots$, respectively.

Definition 1. (see [2]): An uncertain variable is a function $\xi$ from an uncertainty space $(\Gamma, \mathcal{L}, \mathcal{M})$ to the set of real numbers such that $\{\xi \in B\}$ is an event for any Borel set $B$ of real numbers.

Remark 1: Note that the event $\{\xi \in B\}$ is a subset of the universal set $\{\xi \in B\}=\{\gamma \in \Gamma \mid \xi(\gamma) \in$ $B\}$.

Definition 2. (Liu [14]): An uncertainty distribution $\Phi(x)$ is said to be regular if it is a continuous and strictly increasing function with respect to $x$ at which $0<\Phi(x)<1$, and

$$
\lim _{x \rightarrow-\infty} \Phi(x)=0, \lim _{x \rightarrow+\infty} \Phi(x)=1 .
$$

Definition 3. (Liu [14]): Let $\xi$ be an uncertain variable with regular uncertainty distribution $\Phi(x)$. Then the inverse function $\Phi^{-1}(\alpha)$ is called the inverse uncertainty distribution of $\xi$.

Theorem 1. (Liu [14]): Let $\xi_{1}, \xi_{2}, \cdots, \xi_{n}$ be independent uncertain variables with regular uncertainty distributions $\Phi_{1}, \Phi_{2}, \ldots, \Phi_{n}$, respectively. If $f\left(\xi_{1}, \xi_{2}, \cdots, \xi_{n}\right)$ is strictly increasing with respect to $\xi_{1}, \xi_{2}, \cdots, \xi_{m}$ and strictly decreasing with respect to $\xi_{m+1}, \xi_{m+2}, \cdots, \xi_{n}$, then

$$
\xi=f\left(\xi_{1}, \xi_{2}, \cdots, \xi_{n}\right)
$$

has an inverse uncertainty distribution.

$$
\begin{aligned}
\Psi^{-1}(\alpha)= & f\left(\Phi_{1}^{-1}(\alpha), \cdots, \Phi_{m}^{-1}(\alpha),\right. \\
& \left.\Phi_{m+1}^{-1}(1-\alpha), \cdots, \Phi_{n}^{-1}(1-\alpha)\right) .
\end{aligned}
$$

\subsection{Chance theory}

Definition 4. (Liu [1]): Let $(\Gamma, \mathcal{L}, \mathcal{M})$ be an uncertainty space and let $(\Omega, \mathcal{A}, \operatorname{Pr})$ be a probability space. Then the product $(\Gamma, \mathcal{L}, \mathcal{M}) \times(\Omega, \mathcal{A}, \operatorname{Pr})$ is called a chance space

$(\Gamma, \mathcal{L}, \mathcal{M}) \times(\Omega, \mathcal{A}, \operatorname{Pr})=(\Gamma \times \Omega, \mathcal{L} \times \mathcal{A}, \mathcal{M} \times \operatorname{Pr})$.

Definition 5. (see [1]): An uncertain random variable is a function $\xi$ from a chance space $(\Gamma, \mathcal{L}, \mathcal{M}) \times(\Omega, \mathcal{A}, \operatorname{Pr})$ to the set of real numbers such that $\{\xi \in B\}$ is an event in an event in $\mathcal{L} \times \mathcal{A}$ for any Borel set $B$ of real numbers.

Theorem 2. (see [14]): Let $\xi_{1}, \xi_{2}, \cdots, \xi_{n}$ be uncertain random variables on the chance space $(\Gamma, \mathcal{L}, \mathcal{M}) \times(\Omega, \mathcal{A}, \operatorname{Pr})$, and let $f$ be a measurable function. Then

$$
\xi=f\left(\xi_{1}, \xi_{2}, \cdots, \xi_{n}\right)
$$

is an uncertain random variable determined by

$$
\xi(\gamma, \omega)=f\left(\xi_{1}(\gamma, \omega), \xi_{2}(\gamma, \omega), \cdots, \xi_{n}(\gamma, \omega)\right)
$$

for all $(\gamma, \omega) \in \Gamma \times \Omega$.

Theorem 3. (Liu [15]): Let $\eta_{1}, \eta_{2}, \cdots, \eta_{m}$ be independent random variables with probability distributions $\Psi_{1}, \Psi_{2}, \cdots, \Psi_{m}$, respectively, and let $\tau_{1}, \tau_{2}, \cdots, \tau_{n}$ be independent uncertain variables. Assume $f$ is a measurable function. Then the uncertain random variable

$$
\xi=f\left(\eta_{1}, \eta_{2}, \cdots, \eta_{m}, \tau_{1}, \tau_{2}, \cdots, \tau_{n}\right)
$$

has a chance distribution

$$
\begin{gathered}
\Phi(x)=\int_{\Re^{m}} F\left(x ; y_{1}, y_{2}, \cdots, y_{m}\right) \mathrm{d} \Psi_{1}\left(y_{1}\right) \\
\quad \mathrm{d} \Psi_{2}\left(y_{2}\right) \cdots \mathrm{d} \Psi_{m}\left(y_{m}\right) .
\end{gathered}
$$

where $F\left(x ; y_{1}, y_{2}, \cdots, y_{m}\right)$ is the uncertainty distribution of the variable

$$
f\left(y_{1}, y_{2}, \cdots, y_{m}, \tau_{1}, \tau_{2}, \cdots, \tau_{n}\right) .
$$

Definition 6. (Liu [1]): Let $\xi$ be an uncertain random variable. Then its chance distribution is defined by

$$
\Phi(x)=\operatorname{Ch}\{\xi \leq x\}
$$

for any $x \in \Re$.

Theorem 4. Let $\xi$ be an uncertain random variable. Then its expected value is

$$
E[\xi]=\int_{0}^{+\infty} \operatorname{Ch}\{\xi \geq \mathrm{r}\} \mathrm{dr}-\int_{-\infty}^{0} \operatorname{Ch}\{\xi \leq \mathrm{r}\} \mathrm{dr}
$$

provided that at least one of the two integrals is finite. 
Theorem 5. Let $\xi$ be an uncertain random variable with regular chance distribution $\Phi$. If the expected value exists, then

$$
E[\xi]=\int_{0}^{1} \Phi^{-1}(\alpha) \mathrm{d} \alpha .
$$

In order to obtain the chance distribution, we prove following theorems and develop a simulation algorithm.

\section{A simulation algorithm with uncertain random variables}

In this section, by employing the above definition and theorems, the chance distribution will be obtained. To do this, the following theorems is proved and besides a simulation algorithm is developed.

In fact, Formula (3) is a theoretical formula, which is not easy to use in most cases due to the complexity of chance distribution function. To overcome the difficulty, an uncertain random simulation is proposed to evaluate the chance distribution. First, we introduce the concepts of $\alpha$-pessimistic value and $\alpha$-optimistic value for an uncertain random variable. Then, we approximate the chance distribution, $\alpha$-pessimistic value and $\alpha$-optimistic value by using a numerical integration method.

Definition 7. Let $\xi$ be an uncertain random variable on chance space $(\Gamma, \mathcal{L}, \mathcal{M}) \times(\Omega, \mathcal{A}, \operatorname{Pr})$ and $\alpha \in(0,1]$. Then,

$$
\xi_{\text {inf }}(\alpha)=\inf \{r \mid \operatorname{Ch}\{\xi \leq r\} \geq \alpha\}
$$

and

$$
\xi_{\text {sup }}(\alpha)=\sup \{r \mid \operatorname{Ch}\{\xi \geq r\} \geq \alpha\}
$$

are called the $\alpha$-pessimistic value and the $\alpha$-optimistic value of $\xi$, respectively.

Note that Random variables and uncertain variables are special uncertain random variables. The $\alpha$-pessimistic value and the $\alpha$-optimistic value of linear uncertain variable $\mathcal{L}(a, b)$ are $\xi_{\text {inf }}(\alpha)=$ $(1-\alpha) a+\alpha b$ and $\xi_{\text {sup }}(\alpha)=\alpha a+(1-\alpha) b$.

Theorem 6. Let $\xi$ be an ordinary uncertain random variable and $\alpha \in(0,1]$. Then, we have

$$
\operatorname{Ch}\left\{\xi \leq \xi_{\text {inf }}(\alpha)\right\}=\alpha .
$$

Proof. Since the chance distribution is continuous, it follows from the definition of the $\alpha$-pessimistic value for each $\alpha \in(0,1]$, we have $\operatorname{Ch}\left\{\xi \leq \xi_{\text {inf }}(\alpha)\right\}=$ $\lim _{n \rightarrow \infty} \operatorname{Ch}\left\{\xi \leq \xi_{\text {inf }}(\alpha)-1 / \mathrm{n}\right\} \leq \alpha, \quad$ and $\stackrel{\mathrm{n} \rightarrow \infty}{\mathrm{Ch}}\left\{\xi \leq \xi_{\text {inf }}(\alpha)\right\}=\lim _{n \rightarrow \infty} \operatorname{Ch}\left\{\xi \leq \xi_{\text {inf }}(\alpha)+1 / n\right\} \geq$ $\alpha$, which imply that $\operatorname{Ch}\left\{\xi \leq \xi_{\text {inf }}(\alpha)\right\}=\alpha$ holds. The theorem is proved.
Theorem 7. Let $\xi$ be an ordinary uncertain random variable and $\alpha \in(0,1]$. Then, we have

$$
\operatorname{Ch}\left\{\xi \geq \xi_{\text {sup }}(\alpha)\right\}=\alpha .
$$

Proof. Since the continuity of chance distribution, it follows from the definition of the $\alpha$-optimistic value for each $\alpha \in(0,1]$ that $\operatorname{Ch}\left\{\xi \geq \xi_{\text {sup }}(\alpha)\right\}=$ $\lim _{\mathrm{n} \rightarrow \infty} \operatorname{Ch}\left\{\xi \geq \xi_{\text {sup }}(\alpha)-1 / \mathrm{n}\right\} \geq \alpha$ and $\operatorname{Ch}\left\{\xi \geq \xi_{\text {sup }}(\alpha)\right\}=\lim _{n \rightarrow \infty} \operatorname{Ch}\left\{\xi \geq \xi_{\text {sup }}(\alpha)+1 / n\right\} \leq$ $\alpha$, which imply that $\operatorname{Ch}\left\{\xi \geq \xi_{\text {sup }}(\alpha)\right\}=\alpha$ holds. The theorem is proved.

Theorem 8. Let $\xi$ be an uncertain random variable and $\alpha \in(0,1]$. Then, we have

$$
\xi_{\text {inf }}(\alpha)=\Phi^{-1}(\alpha)
$$

Proof. It follows from Definition 7 immediately.

Theorem 9. Let $\xi$ be an uncertain random variable and $\alpha \in(0,1]$. Then, we have

$$
\xi_{\text {inf }}(\alpha)=\xi_{\text {sup }}(1-\alpha) .
$$

Proof. It follows from Equation (8) that

$$
\mathrm{Ch}\left\{\xi \geq \xi_{\text {sup }}(1-\alpha)\right\}=1-\alpha .
$$

Thus,

$$
\begin{aligned}
\operatorname{Ch}\left\{\xi \leq \xi_{\text {sup }}(1-\alpha)\right\} & =1-\operatorname{Ch}\left\{\xi \geq \xi_{\text {sup }}(1-\alpha)\right\} \\
& =1-(1-\alpha) \\
& =\alpha
\end{aligned}
$$

Thus, we have $\xi_{\text {inf }}(\alpha)=\xi_{\text {sup }}(1-\alpha)$. The theorem is proved.

Theorem 10. Let $\xi$ be an uncertain random variable and $\alpha \in(0,1]$. Then, we have

$\xi_{\text {sup }}(\alpha)=\Phi^{-1}(1-\alpha) \quad$ and $\quad \xi_{\text {sup }}(1-\alpha)=\Phi^{-1}(\alpha)$.

Proof. It follows from Theorems 8 and 9 ,

Theorem 11. Let $\xi$ be an ordinary uncertain random variable. Then, we have

$$
E[\xi]=\int_{0}^{1} \xi_{\text {inf }}(\alpha) \mathrm{d} \alpha .
$$

Proof. Since $\Phi(x)$ is strictly increasing and continuous, we get

$$
\begin{aligned}
\xi_{\text {inf }}(\alpha) & =\inf \{\mathrm{r} \mid \operatorname{Ch}\{\xi \leq \mathrm{r}\} \geq \alpha\} \\
& =\inf \{\mathrm{r} \mid \Phi(\mathrm{r}) \leq \alpha\} \\
& =\Phi^{-1}(\alpha) .
\end{aligned}
$$

According to Definition (), we have $E[\xi]=$ $\int_{0}^{1} \xi_{\text {inf }}(\alpha) \mathrm{d} \alpha$. The Theorem is proved. 
Theorem 12. Let $\xi$ be an ordinary uncertain random variable. Then, we have

$$
E[\xi]=\int_{0}^{1} \xi_{\text {sup }}(\alpha) \mathrm{d} \alpha .
$$

Proof. Since $\Phi(x)$ is strictly increasing and continuous, we get

$$
\begin{aligned}
\xi_{\text {sup }}(\alpha) & =\sup \{\mathrm{r} \mid \operatorname{Ch}\{\xi \geq \mathrm{r}\} \geq \alpha\} \\
& =\sup \{\mathrm{r} \mid \Phi(\mathrm{r}) \leq 1-\alpha\} \\
& =\Phi^{-1}(1-\alpha) .
\end{aligned}
$$

Thus, for all $\alpha \in(0,1)$, we have

$$
\begin{aligned}
\int_{0}^{1} \xi_{\text {sup }}(\alpha) \mathrm{d} \alpha & =\int_{0}^{1} \Phi^{-1}(1-\alpha) \mathrm{d} \alpha \\
& =\int_{0}^{1} \Phi^{-1}(\alpha) \mathrm{d} \alpha=\mathrm{E}[\xi]
\end{aligned}
$$

The theorem is proved.

Theorem 13. Let $\xi$ be an ordinary uncertain random variable. Then, we have

$$
E[\xi]=\frac{1}{2} \int_{0}^{1}\left[\xi_{\text {inf }}(\alpha)+\xi_{\text {sup }}(\alpha)\right] \mathrm{d} \alpha .
$$

Proof. It follows directly from Theorem 11 and Theorem 12 .

Theorem 14. Let $\eta_{1}, \eta_{2}, \cdots, \eta_{m}$ be independent random variables with probability distributions $\Psi_{1}, \Psi_{2}, \cdots, \Psi_{m}$, and let $\tau_{1}, \tau_{2}, \cdots, \tau_{n}$ be independent uncertain variables with regular uncertainty distributions $\Upsilon_{1}, \Upsilon_{2}, \cdots, \Upsilon_{n}$. Assume $f\left(\eta_{1}, \eta_{2}, \cdots, \eta_{m}, \tau_{1}, \tau_{2}, \cdots, \tau_{n}\right)$ is strictly increasing with respect to $\tau_{1}, \tau_{2}, \cdots, \tau_{k}$ and strictly decreasing with respect to $\tau_{k+1}, \tau_{k+2}, \cdots, \tau_{n}$. Then

$$
\xi=f\left(\eta_{1}, \eta_{2}, \cdots, \eta_{m}, \tau_{1}, \tau_{2}, \cdots, \tau_{n}\right)
$$

has a chance distribution

$$
\begin{gathered}
\Phi(x)=\int_{\Re^{m}} F\left(x ; y_{1}, y_{2}, \cdots, y_{m}\right) \mathrm{d} \Psi_{1}\left(y_{1}\right) \\
\mathrm{d} \Psi_{2}\left(y_{2}\right) \cdots \mathrm{d} \Psi_{m}\left(y_{m}\right) .
\end{gathered}
$$

where $F\left(x ; y_{1}, y_{2}, \cdots, y_{m}\right)$ is determined by its inverse uncertainty distribution

$$
\begin{gathered}
F^{-1}\left(y_{1}, y_{2}, \ldots, y_{m}, \Upsilon_{1}^{-1}(\alpha), \ldots\right. \\
\left.\Upsilon_{2}^{-1}(\alpha), \ldots, \Upsilon_{n}^{-1}(\alpha)\right) \\
F^{-1}\left(y_{1}, y_{2}, \cdots, y_{m},\left(\xi_{1}\right)_{\sup }(1-\alpha), \cdots\right. \\
\left.\left(\xi_{k}\right)_{\sup }(1-\alpha),\left(\xi_{k+1}\right)_{\sup }(\alpha), \cdots,\left(\xi_{n}\right)_{\sup }(\alpha)\right) .
\end{gathered}
$$

Proof. It follows from Theorem 1 that inverse uncertainty distribution of $F\left(x ; y_{1}, y_{2}, \cdots, y_{m}\right)$ is determined by

$$
\begin{aligned}
& F^{-1}\left(y_{1}, y_{2}, \cdots, y_{m}, \Upsilon_{1}^{-1}(\alpha), \cdots, \Upsilon_{k}^{-1}(\alpha),\right. \\
& \left.\Upsilon_{k+1}^{-1}(1-\alpha), \cdots, \Upsilon_{n}^{-1}(1-\alpha)\right) .
\end{aligned}
$$

According to Theorem 10, we substitute $\Upsilon_{1}^{-1}(\alpha), \cdots, \Upsilon_{k}^{-1}(\alpha)$ with $\quad\left(\xi_{1}\right)_{\text {sup }}(1-$ $\alpha), \cdots,\left(\xi_{k}\right)_{\sup }(1 \quad-\alpha) \quad$ and $\quad \Upsilon_{k+1}^{-1}(1 \quad-$ $\alpha), \cdots, \Upsilon_{n}^{-1}(1-\alpha)$ with $\left(\xi_{k+1}\right)_{\sup }(\alpha), \cdots,\left(\xi_{n}\right)_{\sup }(\alpha)$. Thus, Formula (19) holds. The theorem is completed.

Theorem 15. Let $\eta_{1}, \eta_{2}, \cdots, \eta_{m}$ be independent random variables with probability distributions $\Psi_{1}, \Psi_{2}, \cdots, \Psi_{m}$, and let $\tau_{1}, \tau_{2}, \cdots, \tau_{n}$ be independent uncertain variables with regular uncertainty distributions $\Upsilon_{1}, \Upsilon_{2}, \cdots, \Upsilon_{n}$. Assume $f\left(\eta_{1}, \eta_{2}, \cdots, \eta_{m}, \tau_{1}, \tau_{2}, \cdots, \tau_{n}\right)$ is strictly increasing with respect to $\tau_{1}, \tau_{2}, \cdots, \tau_{k}$ and strictly decreasing with respect to $\tau_{k+1}, \tau_{k+2}, \cdots, \tau_{n}$. Then

$$
\xi=f\left(\eta_{1}, \eta_{2}, \cdots, \eta_{m}, \tau_{1}, \tau_{2}, \cdots, \tau_{n}\right)
$$

has a chance distribution

$$
\begin{gathered}
\Phi(x)=\int_{\Re^{m}} F\left(x ; y_{1}, y_{2}, \cdots, y_{m}\right) \mathrm{d} \Psi_{1}\left(y_{1}\right) \\
\mathrm{d} \Psi_{2}\left(y_{2}\right) \cdots \mathrm{d} \Psi_{m}\left(y_{m}\right) .
\end{gathered}
$$

where $F\left(x ; y_{1}, y_{2}, \cdots, y_{m}\right)$ is determined by its inverse uncertainty distribution

$$
\begin{gathered}
F^{-1}\left(y_{1}, y_{2}, \ldots, y_{m}, \Upsilon_{1}^{-1}(\alpha), \ldots,\right. \\
\left.\Upsilon_{2}^{-1}(\alpha), \ldots, \Upsilon_{n}^{-1}(\alpha)\right) \\
F^{-1}\left(y_{1}, y_{2}, \ldots, y_{m},\left(\xi_{1}\right)_{\mathrm{inf}}(\alpha), \cdots,\left(\xi_{k}\right)_{\mathrm{inf}}(\alpha),\right. \\
\left.\left(\xi_{k+1}\right)_{\mathrm{inf}}(1-\alpha), \cdots,\left(\xi_{n}\right)_{\mathrm{inf}}(1-\alpha)\right) .
\end{gathered}
$$

Proof. The proof is similar to that of Theorem 14

According to Theorems 1, 17, 8, 9] and 10, we design the following uniform discretization algorithm to simulate $\Phi(x), \alpha$-pessimistic value and $\alpha$-optimistic value. The presented algorithm is very flexible. Because it can even simulate the empirical distribution.

\section{Algorithm 1 (Uniform Discretization Algo- rithm)}

Step 1. Discretize the range of the random variable $\eta_{i}$ into $N_{i}$ equally spaced points.

Step 2. Discretize $\alpha$ into $K$ equally spaced points.

Step 3. Calculate $F^{-1}\left(\alpha ; y_{1}, y_{2}, \cdots, y_{m}\right)$.

Step 4. Calculate $F\left(x ; y_{1}, y_{2}, \cdots, y_{m}\right)=$

$$
\left\{\begin{array}{lll}
0 & \text { if } \quad x \leq x_{1}, \\
\alpha_{i}+\left(\alpha_{i+1}-\alpha_{i}\right) \frac{x-x_{i}}{x_{i+1}-x_{i}} & \text { if } \quad x_{i} \leq x \leq x_{i+1}, \\
& \text { if } \quad x \geq x_{K},
\end{array}\right.
$$

Step 5. Apply numerical integration to compute $\Phi(x), \alpha$-pessimistic value and $\alpha$-optimistic value. 
The uniform discretization algorithm is illustrated by the following example.

\section{A numerical example}

Example Suppose that $\eta_{1}$ and $\eta_{2}$ are independent random variables with probability distributions $U(1,2)$ and $U(2,4)$, and suppose that $\tau_{1}$ and $\tau_{2}$ are independent uncertain variables with uncertainty distributions $\mathcal{L}(1,5)$ and $\mathcal{L}(1,3)$. Then $\xi=\eta_{1}+\eta_{2}+\tau_{1}-\tau_{2}$ is an uncertain random variable. Assume that $\xi$ has chance distribution $\Phi(x)$.

For the sake of simplicity, we set $N_{1}=10, N_{2}=$ $10, K=10$. We also can assign a large number to $N_{1}, N_{2}$ and $K$. The probability distribution function of $\eta_{1}$ and $\eta_{2}$ are

$$
\begin{gathered}
\Psi_{1}\left(y_{1}\right)=\left\{\begin{array}{lll}
0 & \text { if } y_{1} \leq 1, \\
y_{1}-1 & \text { if } 1 \leq y_{1} \leq 2, \text { and } \\
1 & \text { if } y_{1} \geq 2,
\end{array}\right. \\
\Psi_{2}\left(y_{2}\right)=\left\{\begin{array}{lll}
0 & \text { if } y_{2} \leq 2, \\
\frac{y_{2}-2}{2} & \text { if } 2 \leq y_{2} \leq 4, \\
1 & \text { if } & y_{2} \geq 4 .
\end{array}\right.
\end{gathered}
$$

Then, we can have discrete forms of $\Psi_{1}\left(y_{1}\right)$ and $\Psi_{1}\left(y_{2}\right)$ in which $y_{1}=1+0.1 \cdot i$ and $y_{2}=2+0.2 \cdot j$ for $i=1,2, \cdots, 10, j=1,2, \cdots, 10$. The inverse uncertainty distribution function of $\tau_{1}$ and $\tau_{2}$ are $\left(\tau_{1}\right)_{\text {inf }}(\alpha)=(1-\alpha) \cdot 1+\alpha \cdot 5=1+4 \cdot \alpha$ and $\left(\tau_{2}\right)_{\text {inf }}(1-\alpha)=\alpha \cdot 1+(1-\alpha) \cdot 3=3-2 \cdot \alpha$.

The chance distribution of $\xi$ is

$$
\Phi(x)=\frac{1}{2} \int_{2}^{4} \int_{1}^{2} F\left(x ; y_{1}, y_{2}\right) \mathrm{d} y_{1} \mathrm{~d} y_{2}
$$

where $F\left(x ; y_{1}, y_{2}\right)$ is obtained by the inverse uncertainty distribution function $F^{-1}\left(\alpha ; y_{1}, y_{2}\right)=$ $y_{1}+y_{2}+(1+4 \cdot \alpha)-(3-2 \cdot \alpha)$ for each $\alpha \in(0,1]$. This implies that $F^{-1}\left(\alpha ; y_{1}, y_{2}\right)=x_{k}, 1 \leq k \leq 10$ for each $\alpha \in(0,1]$. The value of $F^{-1}\left(\alpha ; y_{1}, y_{2}\right)$ is listed on the Table I.

Then, according to Step 4 , we obtain $F\left(x ; y_{1}, y_{2}\right)$. Now, we get the chance distribution function of $\xi$.

$$
\begin{aligned}
\Phi(x) & =\frac{1}{2} \int_{2}^{4} \int_{1}^{2} F\left(x ; y_{1}, y_{2}\right) \mathrm{d} y_{1} \mathrm{~d} y_{2} \\
& =\frac{1}{2} \sum_{i=1}^{10} \sum_{j=1}^{10} F(x ; 1+0.1 \cdot i, 2+0.2 \cdot j) \cdot 0.1 \cdot 0.2
\end{aligned}
$$

Applying the above presented algorithm, the chance distribution of $\xi$ is obtained as (Figure 1): $\xi_{\text {inf }}(0.2)=3.9$ and $\xi_{\text {sup }}(0.2)=7.4$.

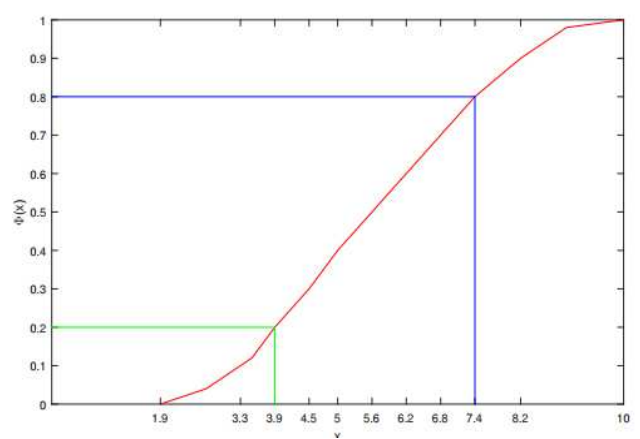

Figure 1. The chance distribution of $\xi$.

\section{Conclusion}

Here, an uncertain random simulation algorithm is presented to illustrate chance distribution. The presented simulation method can be generally applied to uncertain random optimization by approximating chance constraints. The results obtained show that the presented algorithm is the successful for the uncertain random simulation.

\section{Acknowledgments}

The author would like to thank the anonymous reviewers for their valuable comments.

\section{References}

[1] Liu, Y. (2013). Uncertain random variables: A mixture of uncertainty and randomness. Soft Computing, $17(4), 625-634$.

[2] Liu, B. (2007). Uncertainty theory, 2nd ed., SpringerVerlag, Berlin, Germany.

[3] Gao, J. (2013). Uncertain bimatrix game with applications. Fuzzy Optimization and Decision Making, 12(1), 65-78.

[4] Yang, X., and Gao, J. (2016). Linearquadratic uncertain differential game with application to resource extraction problem. IEEE Transactions on Fuzzy Systems, 24(4), 819-826.

[5] Gao, Y., and Qin, Z. (2016) On computing the edgeconnectivity of an uncertain graph. IEEE Transactions on Fuzzy Systems, 24(4), 981-991.

[6] Dalman, H. (2018). Uncertain programming model for multi-item solid transportation problem. International Journal of Machine Learning and Cybernetics, 9(4), 559-567.

[7] Dalman, H. (2018). Uncertain random programming models for fixed charge multi-item solid transportation problem, New Trends in Mathematical Sciences, 6(1), 37-51.

[8] Liu, B. (2014). Uncertain random graph and uncertain random network. Journal of Uncertain Systems, 8(1), 3-12.

[9] Zhou, J., Yang, F., and Wang, K. (2014). Multiobjective optimization in uncertain random environments. Fuzzy Optimization and Decision Making, 13(4), 397-413.

[10] Ahmadzade, H., Gao, R., and Zarei, H. (2016). Partial quadratic entropy of uncertain random variables. Journal of Uncertain Systems, 10(4), 292-301. 
[11] Ke, H., Liu, H., and Tian, G. (2015). An uncertain random programming model for project scheduling problem. International Journal of Intelligent Systems, 30(1), 66-79.

[12] Sheng, Y., and Gao, Y. (2016). Shortest path problem of uncertain random network. Computers and Industrial Engineering, 99, 97-105.

[13] Liu, B. (2009). Some research problems in uncertainty theory. Journal of Uncertain Systems, 3(1), 3-10.

[14] Liu, B. (2010). Uncertainty Theory: A Branch of Mathematics for Modeling Human Uncertainty, Springer-Verlag, Berlin, Germany.
[15] Liu, Y. (2013). Uncertain random programming with applications. Fuzzy Optimization and Decision Making, 12(2), 153-169.

Hasan Dalman received the Ph.D. degree in Mathematical Engineering from Yildiz Technical University in 2015. He is currently assistant professor in the Department of Computer Engineering, Istanbul Gelisim University, Turkey. His current research interests include fuzzy systems, uncertainty theory and mathematical optimization.

An International Journal of Optimization and Control: Theories \& Applications (http://ijocta.balikesir.edu.tr)

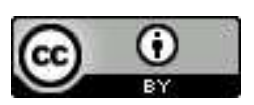

This work is licensed under a Creative Commons Attribution 4.0 International License. The authors retain ownership of the copyright for their article, but they allow anyone to download, reuse, reprint, modify, distribute, and/or copy articles in IJOCTA, so long as the original authors and source are credited. To see the complete license contents, please visit http://creativecommons.org/licenses/by/4.0/. 